\title{
Chronologic changes in P-wave characteristics after the Fontan procedure: The effect of surgical modification
}

\author{
Masahiro Koh, MD, ${ }^{\text {a,b }}$ Hideki Uemura, MD, ${ }^{\text {a,b }}$ Akiko Kada, MPH, ${ }^{\mathrm{c}}$ Koji Kagisaki, MD, ${ }^{\mathrm{a}}$ \\ Ikuo Hagino, $\mathrm{MD},{ }^{\mathrm{a}}$ and Toshikatsu Yagihara, $\mathrm{MD}^{\mathrm{a}}$
}

Objectives: The Fontan-type procedure has undergone 2 major modifications, including intra-atrial baffling and
extracardiac conduit. To clarify the effect of these modifications on arrhythmia propensity, we analyzed chrono-
logic changes in P-wave characteristics after atriopulmonary connection, intra-atrial baffling, or extracardiac con-
duit. Methods: A retrospective analysis was conducted on electrocardiographic data from 40 patients with tricuspid
atresia who underwent the Fontan-type procedure and follow-up for greater than 5 years: 18 had atriopulmonary
connection, 13 had intra-atrial baffling, and 9 had extracardiac conduit. The mean follow-up period in years was
19.8 for atriopulmonary connection, 13.3 for intra-atrial baffling, and 8.0 for extracardiac conduit. We analyzed
chronologic changes in P-wave duration, dispersion, and amplitude and prevalence of sinus node dysfunction.

Results: Atrial tachyarrhythmia was documented in 9 patients with atriopulmonary connection but not in any patients with extracardiac conduit or intra-atrial baffling. Both P-wave maximum duration and dispersion decreased slightly over time with extracardiac conduit but increased progressively in the intra-atrial baffling and atriopulmonary connection groups. Intra-atrial baffling resulted in significantly shorter P-wave duration than atriopulmonary connection, whereas extracardiac conduit had significantly shorter P-wave duration and smaller dispersion than atriopulmonary connection and intra-atrial baffling. P-wave amplitude decreased markedly immediately after surgical intervention with intra-atrial baffling and extracardiac conduit but remained unchanged in patients undergoing atriopulmonary connection. Sinus node dysfunction was found commonly in all 3 groups.

Conclusion: After intra-atrial baffling, patients increasingly had prolonged P-wave duration and larger dispersion associated with sinus node dysfunction, suggesting a propensity to arrhythmia, although less progressive than seen in those undergoing atriopulmonary connection. In contrast, despite an equal prevalence of sinus node dysfunction after extracardiac conduit, the lack of important changes in P-wave characteristics over time suggests that the extracardiac conduit procedure is the preferred option for optimal rhythm prognosis. (J Thorac Cardiovasc Surg 2010;140:137-43)

P-wave characteristics have been shown to reflect structural and electrical remodeling of the atrium. In particular, 2 simple indices, $\mathrm{P}$-wave duration and $\mathrm{P}$-wave dispersion, are established as useful markers for predicting atrial tachyarrhythmia in various clinical settings. ${ }^{1-4}$ A prolonged $\mathrm{P}$-wave duration is known to be associated with fragmented and prolonged atrial activation that could provide the substrate for re-entrant atrial tachyarrhythmia. Similarly, P-wave dispersion, defined as the difference between maximum and minimum P-wave duration recorded from multiple surface

\footnotetext{
From the Departments of Cardiovascular Surgery ${ }^{\mathrm{a}}$ and Clinical Research and Development, ${ }^{\mathrm{c}}$ National Cardiovascular Center, Osaka, Japan, and the Cardiac Division, ${ }^{\mathrm{b}}$ Royal Brompton Hospital, London, United Kingdom.

Disclosures: None.

Read at the Eighty-ninth Annual Meeting of The American Association for Thoracic Surgery, Boston, Mass, May 9-13, 2009.

Received for publication May 3, 2009; revisions received Jan 12, 2010; accepted for publication March 13, 2010; available ahead of print April 29, 2010.

Address for reprints: Masahiro Koh, MD, Department of Cardiac Surgery, Guy's \& St. Thomas' Hospital, Westminster Bridge Road, London SE1 7EH, United Kingdom (E-mail: masahiro.koh@nifty.com).

$0022-5223 / \$ 36.00$

Copyright (c) 2010 by The American Association for Thoracic Surgery

doi:10.1016/j.jtcvs.2010.03.014
}

electrocardiograms, has been reported to be significantly greater in patients with atrial tachyarrhythmia, with this increased dispersion indicating nonuniform, anisotropic, and inhomogeneous atrial conduction. ${ }^{1}$ We have previously investigated the chronologic changes in P-wave characteristics after the classic atriopulmonary connection (APC) type of Fontan procedure and demonstrated that, late after the procedure, patients commonly had prolonged $\mathrm{P}$-wave duration and larger P-wave dispersion before the onset of atrial tachyarrhythmia. ${ }^{5}$

The purpose of this study was to conduct a consecutive review of 12-lead electrocardiograms and 24-hour Holter recordings after either APC, intra-atrial baffling (IB), or extracardiac conduit (EC) Fontan modifications and to compare these among the 3 groups. This review will clarify the effect of these surgical modifications on P-wave characteristics, sinus node function, and rhythm prognosis.

\section{MATERIALS AND METHODS \\ Study Population}

The primary enrollment criterion for this study was either simple tricuspid atresia or stenosis in the setting of situs solitus. In addition, patients with less than 5 years of follow-up were excluded. Between October 1979 and 


\section{Abbreviations and Acronyms \\ $\mathrm{APC}=$ atriopulmonary connection \\ $\mathrm{EC}=$ extracardiac conduit \\ $\mathrm{IB}=$ intra-atrial baffling}

December 2001, a total of 245 patients underwent the Fontan procedure at the National Cardiovascular Center, with 64 having tricuspid atresia or severe tricuspid stenosis in the setting of situs solitus. Fifty-six patients survived and were discharged, with 3 of these survivors dying within 5 years of surgical intervention. Twelve patients had less than 5 years of followup. One patient who had a catheter ablation for Wolff-Parkinson-White syndrome before the Fontan procedure was excluded, resulting in $40 \mathrm{pa}-$ tients being included in the review of the clinical and electrocardiographic data. Because this was a single-center retrospective study with anonymous data, individual informed consent was waived.

The series was divided into 3 cohorts based on the surgical procedure. APC was used in the early era, and then in 1987, we adopted total cavopulmonary connection by means of IB, followed by EC in 1996. Therefore of the 40 patients enrolled in this study, 18 were in the APC group, 13 were in the IB group, and 9 were in the EC group. The 18 patients in the APC group were also involved in our previous study, with all data being re-evaluated and updated. ${ }^{5}$

The preoperative clinical characteristics of the 3 groups are summarized in Table 1. The mean age at the time of the Fontan procedure was $5.3 \pm 4.8$ years for the APC group, $3.9 \pm 2.1$ years for the IB group, and $1.3 \pm 0.4$ years for the EC group. There were significant differences in age and body weight at the time of the Fontan procedure among the 3 groups but no significant differences in the other preoperative parameters. All the patients were in sinus rhythm before the surgical procedures. Fourteen patients undergoing APC, 13 patients undergoing IB, and 5 patients undergoing EC had prior palliative procedures. One patient in the APC group underwent a classic Glenn anastomosis, and 2 patients in the IB group underwent staging bidirectional cavopulmonary anastomosis. Therefore a single-staged Fontan procedure was performed in 17 patients in the APC group, 11 patients in the IB group, and 9 patients in the EC group.

The mean duration of follow-up was $19.8 \pm 4.5$ years (range, 10-27 years) in the APC group, $13.3 \pm 1.3$ years (range, $11-15$ years) in the IB group, and $8.0 \pm 1.5$ years (range, $6-10$ years) in the EC group. Annual follow-up was complete in all but 2 of the patients undergoing APC; 1 patient withdrew from routine follow-up 10 years after the initial Fontan procedure, and the other had less frequent follow-up.

\section{Surgical Techniques}

In the early era, 18 patients underwent the APC Fontan procedure. Fontan circulation was established by means of direct anastomosis between the pulmonary artery and the right atrial appendage, and closure of the intraatrial communication was established by means of direct suturing or the patch technique. ${ }^{5}$

In 1987, we introduced total cavopulmonary connection using IB, which consists of a longitudinal right atrial incision and construction of a polytetrafluoroethylene or heterologous pericardial baffle connecting the inferior caval vein along the anterior wall to the tip of the right atrial appendage into the pulmonary artery. In contrast with the original lateral tunnel procedure, a patch was placed anteriorly so that the sinus node was not only at a minimum risk of surgical injury but was also placed within the low-pressure atrial chamber, with the aim of minimizing future sinus node dysfunction. ${ }^{6}$ A bidirectional cavopulmonary anastomosis was constructed at the same time in all patients, with the exception of 2 patients who had a previous bidirectional cavopulmonary anastomosis.

We introduced the EC Fontan-type procedure in 1996. EC involves separating the inferior caval vein from the right atrium and anatomizing an ap- propriately sized conduit in an end-to-end fashion with the inferior vena cava. The conduit is then anastomosed to the underside of the pulmonary arteries. The conduit consisted of an autologous pericardial roll in 5 patients and an 18 -mm polytetrafluoroethylene graft in 1 patient. ${ }^{7}$ In the other 2 patients, the inferior caval vein was connected directly to the pulmonary artery without a conduit. ${ }^{8}$ Cardiopulmonary bypass was not used in 6 patients. ${ }^{9}$ A bidirectional cavopulmonary anastomosis was constructed at the same time in all the patients.

Concomitant procedures were required in 6 patients. One patient in the APC group with a previous Glenn anastomosis underwent reconstruction of the confluent pulmonary arteries, 2 patients in the IB group and 1 patient in the EC group underwent the Damus-Kay-Stansel procedure, and the other 2 patients in the EC group underwent resection of subaortic stenosis. No fenestration was created in any of the 3 groups.

\section{Analysis of Electrocardiographic Markers}

Consecutive 12-lead surface electrocardiograms were examined retrospectively in all 40 patients from the early preoperative period to the latest follow-up. The mean number of electrocardiograms per patient included in this analysis was $15.7 \pm 8.1$ in the APC group, $14.4 \pm 3.6$ in the IB group, and $8.2 \pm 2.2$ in the EC group. A total of 543 electrocardiograms were investigated. The methods for measurement of electrocardiographic markers were the same as described in our previous report. ${ }^{5}$ Briefly, we measured P-wave duration, dispersion, and amplitude simultaneously in all 12 leads. Maximum and minimum P-wave durations in all 12 electrocardiographic leads were then calculated. P-wave dispersion was defined as the difference between the maximum and minimum P-wave durations of an individual electrocardiogram, ${ }^{1}$ whereas $\mathrm{P}$-wave amplitude was measured in the lead II as the absolute value from the isometric baseline to positive and negative maximum deflection. Biphasic P-wave amplitude was defined as the sum of both the absolute values of positive and negative deflections. ${ }^{10}$

\section{Sinus Node Dysfunction}

All 12-lead electrocardiograms and 24-hour Holter recordings were reviewed. The 24-hour Holter recordings were recorded at least every 5 years during the follow-up period in all the patients except for 1 in the IB group and 1 in the EC group. The mean number of Holter recordings included in this analysis was $6.4 \pm 3.5$ in the APC group, $4.9 \pm 3.2$ in the IB group, and $2.0 \pm 1.1$ in the EC group. In accordance with previously established criteria, sinus node dysfunction was diagnosed when more than 1 of the following 3 characteristics were found in either the 12-lead electrocardiograms or Holter recordings: (1) the presence of junctional rhythm, (2) a sinus pause duration of 3 seconds or greater with or without an escape rhythm, or (3) bradycardia of 2 standard deviations or more below the age-adjusted heart rate without the use of antiarrhythmic drugs. ${ }^{11-15}$ The time to sinus node dysfunction was defined as the time to the first episode documented after the immediate postoperative period.

\section{Data Analysis}

The continuous variables are expressed as the mean \pm 1 standard deviation. The cumulative event-free rate was estimated by using the Kaplan-Meier method. Comparison of patient characteristics among the 3 groups was carried out with either the Kruskal-Wallis test or ANOVA, as appropriate. Changes in electrocardiographic markers were analyzed by using a general linear mixed model, with years as the fixed effect and patients as the random effect. When a patient had more than 1 measurement within a year, the value of the first measurement was used. All statistical tests were conducted with SAS Statistical Software version 8.02 (SAS Institute Inc, Cary, NC).

\section{RESULTS}

\section{Arrhythmia}

No patient in any of the 3 groups died during the followup period. Although no patient in the IB or EC groups had an 
TABLE 1. Preoperative clinical characteristics and P-wave characteristics

\begin{tabular}{|c|c|c|c|c|}
\hline & $\operatorname{APC}(n=18)$ & IB $(\mathbf{n}=13)$ & $\mathbf{E C}(\mathbf{n}=9)$ & $P$ value \\
\hline Age (y) & $5.3 \pm 4.8$ & $3.9 \pm 2.1$ & $1.3 \pm 0.4$ & .001 \\
\hline Weight $(\mathrm{kg})$ & $16.6 \pm 9.3$ & $12.6 \pm 3.9$ & $8.8 \pm 1.8$ & .001 \\
\hline $\mathrm{SaO}_{2}(\%)$ & $81.8 \pm 7.2$ & $80.8 \pm 4.7$ & $79.1 \pm 1.7$ & .577 \\
\hline Mean PAP $(\mathrm{mm} \mathrm{Hg})$ & $12.4 \pm 4.4$ & $15.0 \pm 5.4$ & $16.3 \pm 3.9$ & .120 \\
\hline $\mathrm{Qp} / \mathrm{Qs}$ & $1.72 \pm 0.75$ & $1.48 \pm 0.63$ & $1.43 \pm 0.39$ & .526 \\
\hline LVEDV $(\%)$ & $203.9 \pm 57.0$ & $199.0 \pm 47.3$ & $174.2 \pm 27.9$ & .333 \\
\hline $\operatorname{LVEF}(\%)$ & $50.8 \pm 15.8$ & $64.5 \pm 10.7$ & $63.9 \pm 7.9$ & .356 \\
\hline Heart rate (beats/min) & $107.2 \pm 4.6$ & $123.4 \pm 5.6$ & $118.8 \pm 6.5$ & .077 \\
\hline Maximal P-wave duration (ms) & $111.6 \pm 3.4$ & $115.0 \pm 4.2$ & $112.0 \pm 4.8$ & .805 \\
\hline P-wave dispersion (ms) & $43.1 \pm 2.6$ & $51.3 \pm 3.2$ & $42.7 \pm 3.7$ & .110 \\
\hline P-wave amplitude (mV) & $2.52 \pm 0.26$ & $3.27 \pm 0.33$ & $3.03 \pm 0.36$ & .193 \\
\hline P-wave axis & $38.3 \pm 3.8$ & $42.0 \pm 4.5$ & $36.8 \pm 5.4$ & .733 \\
\hline
\end{tabular}

$A P C$, Atriopulmonary connection; $I B$, intra-atrial baffling; $E C$, extracardiac conduit; $P A P$, pulmonary artery pressure; $Q p / Q s$, pulmonary/systemic blood flow ratio, $L V E D V$, left ventricular end-diastolic volume; $L V E F$, left ventricular ejection fraction.

atrial tachyarrhythmia, 9 patients in the APC group had this condition, as described in detail in our previous report. ${ }^{5}$ Freedom from atrial tachyarrhythmia in the APC group was $88.5 \% \pm 7.6 \%$ at 10 years, $65.0 \% \pm 11.6 \%$ at 15 years, and $41.2 \% \pm 13.2 \%$ at 20 years (Figure $1, A$ ). Twelve patients in the APC group underwent conversion to total cavopulmonary connection with antiarrhythmic surgery, and 1 patient underwent catheter ablation, as described

Freedom from Arrhythmia

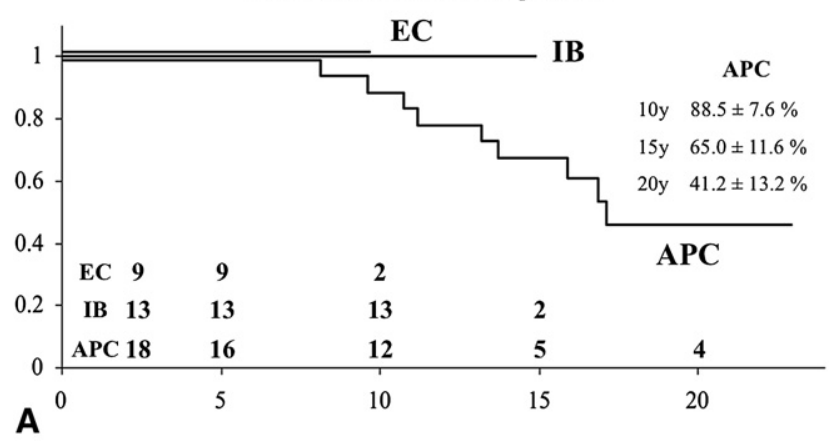

Freedom from Sinus Node Dysfunction

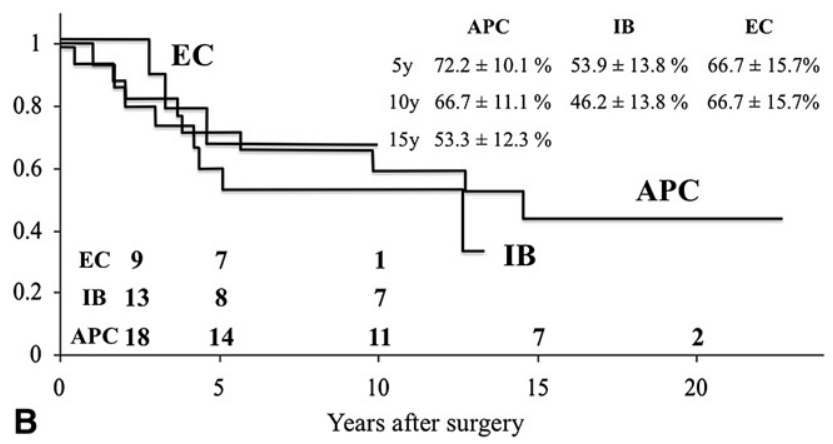

FIGURE 1. A, Freedom from atrial tachyarrhythmia. The cumulative event-free rate was estimated by using the Kaplan-Meier method. B, Freedom from sinus node dysfunction. The cumulative event-free rate was estimated by using the Kaplan-Meier method. $A P C$, atriopulmonary connection; $I B$, intra-atrial baffling; $E C$, extracardiac conduit. previously. ${ }^{5}$ No patient in the IB and EC groups required permanent pacemaker implantation, whereas 2 patients in the APC group underwent such an implantation after the conversion procedure.

\section{P-Wave Characteristics}

There were no significant differences in preoperative $\mathrm{P}$-wave characteristics among the 3 groups (Table 1). Maximum P-wave duration increased progressively over time in the patients with APC and IB but decreased slightly in the patients with EC (Figure 2). The patients with IB had significantly shorter maximum $\mathrm{P}$-wave durations than the patients with APC $(P=.001)$. The patients with EC also had significantly shorter maximum $\mathrm{P}$-wave durations than the patients with $\operatorname{APC}(P<.001)$ and the patients with $\operatorname{IB}(P=.011)$. Similarly, P-wave dispersion increased progressively over time in the APC and IB groups but decreased marginally in the EC group. There was no significant difference in $\mathrm{P}$-wave dispersion between the APC and IB groups, whereas the EC group had significantly shorter P-wave dispersions than the APC $(P=.004)$ and IB $(P=.022)$ groups. $\mathrm{P}$-wave amplitude remained unchanged for a period in the APC group and then decreased gradually approximately 15 years after surgical intervention. In contrast, in the IB and EC groups P-wave amplitude decreased dramatically immediately after surgical intervention and subsequently remained at these low levels.

\section{Sinus Node Dysfunction}

None of the 3 groups had sinus node dysfunction at the time of hospital discharge. Sinus node dysfunction was observed in 20 patients during follow-up: 9 in the APC group, 8 in the IB group, and 3 in the EC group (Figure 1, $B$ ). All of these patients had bradycardia with a heart rate of 2 standard deviations or greater below the age-adjusted heart rate, with the exception of 1 patient with EC who had a frequent junctional rhythm. There was no significant difference in the prevalence of sinus node dysfunction among the 3 groups. 


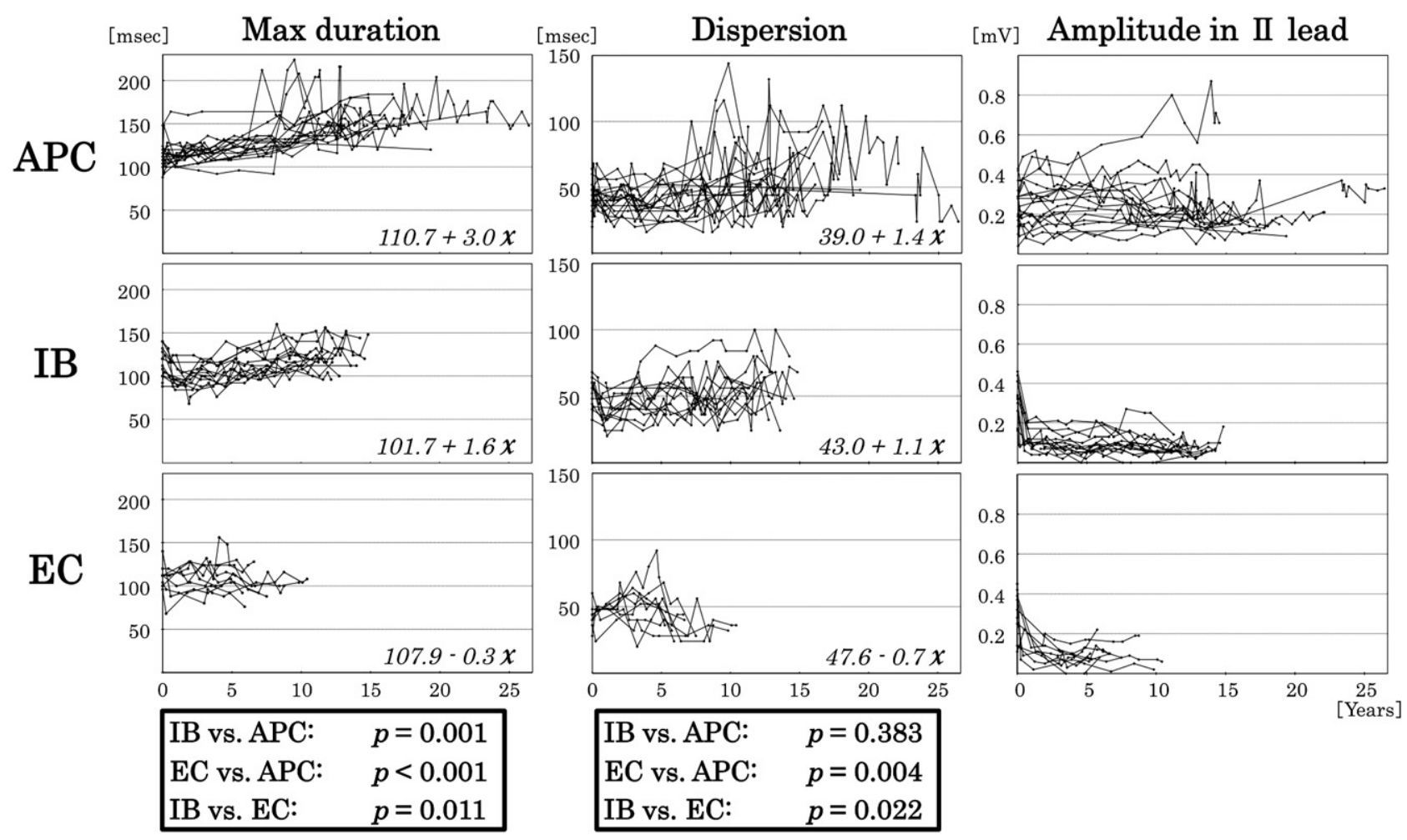

FIGURE 2. Chronologic changes in $\mathrm{P}$-wave characteristics. Changes in maximum $\mathrm{P}$-wave duration and $\mathrm{P}$-wave dispersion were analyzed with the general linear mixed model, with years as a fixed effect and patients as a random effect. $x$, Years after surgical intervention. APC, Atriopulmonary connection; $I B$, intra-atrial baffling; $E C$, extracardiac conduit.

\section{DISCUSSION}

The occurrence of atrial tachyarrhythmias as a consequence of complex atrial scars and abnormal atrial wall stress after APC was the predominant reason for modification of the Fontan procedure. This led to the development of the IB procedures, such as the lateral tunnel procedure. ${ }^{16}$ However, this technique involves extensive suture lines in the atrium and has the potential to cause atrial distension, which might lead to sinus node dysfunction, atrial tachyarrhythmia, or both, even when the part of the atrium placed under high pressure is limited. ${ }^{17}$ In contrast, the EC procedure, the most recent modification of the technique, leaves the entire atrium at low pressure and avoids significant suture lines. Theoretically, the EC procedure should therefore have a lower risk for sinus node dysfunction and atrial tachyarrhythmia. The IB and EC procedures have been shown to reduce the incidence of atrial tachyarrhythmia compared with the APC procedure ${ }^{18}$; however, detailed electrocardiographic investigations are still scarce.

We showed previously that there was a progressive increase in both P-wave duration and dispersion over time after the APC Fontan procedure, which preceded the onset of symptomatic atrial tachyarrhythmia. ${ }^{5}$ Although less prominent than the APC procedure, a similar trend was noticed in the patients undergoing IB. Because this study is a chronologic analysis of changes in P-wave characteristics over a long period since childhood, age-related influences on P-wave characteristics need to be taken into consideration. It has been reported that $\mathrm{P}$-wave duration correlates with age because of an age-related delay in atrial conduction. ${ }^{19}$ However, the increase in P-wave duration we observed over time in the APC and IB groups was obviously more than an age-related increase. Wong and colleagues ${ }^{4}$ suggested that a $\mathrm{P}$-wave duration of greater than $134 \mathrm{~ms}$ and a P-wave dispersion of greater than $66 \mathrm{~ms}$ had good sensitivity and specificity for identifying patients undergoing the Fontan procedure with a history of atrial tachyarrhythmia. The majority of patients in the APC and IB groups were approaching these criteria late after the Fontan procedure. On the basis of this finding, it is highly probable that the patients in the IB group had a predisposition to atrial tachyarrhythmia, although not as progressive as seen in the APC group. In clear contrast, there was a decreasing tendency in both P-wave duration and dispersion in the EC group, which suggests that patients in the EC group are less prone to atrial tachyarrhythmia.

There was an intriguing difference in the change in P-wave amplitude between the IB/EC and APC groups. In the APC group the P-wave amplitude remained unchanged and then gradually decreased approximately 15 years after 
surgical intervention. Li and colleagues ${ }^{20}$ reported that a progressive broadening of $\mathrm{P}$-wave duration and a decrease in its amplitude commonly preceded the occurrence of atrial tachyarrhythmia in patients with adult congenital heart disease. Our findings in the APC group are consistent with their findings. On the other hand, the IB and EC groups had a completely different pattern because the P-wave amplitude decreased dramatically after surgical intervention and remained low. Kavey and coworkres ${ }^{13}$ reported similar findings in a study of patients after the lateral tunnel Fontan procedure and speculated that this decrease in amplitude might have been related to alterations in conduction in the presence of the intra-atrial baffle and extensive suture lines. However, in the present study this trend was present not only in the IB group but also in the EC group, where the atrial suture line should be minimal. It can be argued that this different pattern in P-wave amplitude between the APC and IB/EC groups might be related to the degree of pressure load, volume load, or both to the atrium; however, the data in this study did not address this possibility.

This study demonstrates that patients who undergo either the IB or EC Fontan procedure can have a significant incidence of sinus node dysfunction, although none of these patients required any treatment during follow-up. Sinus node dysfunction after the Fontan-type operation is generally considered to result from direct injury to the sinus node, its blood supply, or both. Accordingly, we made a technical modification to the IB procedure to avoid injury to the sinus node. We used the anterior wall of the atrium as the Fontan pathway and placed the sinus node within the low-pressure atrial chamber to avoid chronic pressure load to the sinus node. ${ }^{6}$ In the EC procedure, which is our current preference, the risk of damage to the sinus node should be theoretically minimal. However, contrary to our expectations, we could not show any advantage of these 2 surgical modifications over the APC procedure for maintaining healthy sinus node function. The incidence of sinus node dysfunction after the Fontan-type procedure might also be affected by the technique used for the superior cavopulmonary connections. All of the patients in the IB and EC groups in this series had a bidirectional cavopulmonary anastomosis. The performance of this anastomosis might result in manipulation of the sinus node or impairment of its arterial supply, such that the patient is at increased risk of sinus node dysfunction, although this risk should be less than that for the hemiFontan procedure. ${ }^{14,15}$ Another possible explanation for surgical damage to the sinus node is repeated dissections around the sinus node during multiple surgical procedures. ${ }^{14}$ However, in this particular series 37 of the 40 patients had the Fontan procedure in a single-staged fashion, and therefore repeated dissection was unlikely to be a contributing factor to sinus node dysfunction. With regard to sinus node dysfunction related to surgical damage, patients typically have sinus node dysfunction in the early postoperative period. ${ }^{14,15}$ In this study sinus node dysfunction was not observed in any of the 3 groups during the early postoperative period. This finding might be inconsistent with the hypothesis that in our study population surgical damage to the sinus node was a dominant mechanism predisposing to late sinus node dysfunction. Sinus node dysfunction can also be caused by impairment of cardiac autonomic nervous activity. In all types of the Fontan procedure, there is some unavoidable damage to cardiopulmonary nerves around the vessels and atrium. ${ }^{21}$ Some investigators have suggested that, in addition to surgical damage, specific hemodynamic changes in the Fontan circulation, characterized by low cardiac output and high venous pressure, might be responsible for the diminished autonomic nervous activity. ${ }^{22}$

The clinical implication of sinus node dysfunction that occurs late in patients after the Fontan procedure is controversial. Blaufox and associates ${ }^{23}$ reported that bradycardia, although common, was not associated with decreased functional status in this setting. They speculated that a low resting heart rate might confer mechanical advantages to the Fontan ventricle in the context that longer diastolic filling times support the preload dependency of the Fontan circulation or improve load-independent diastolic mechanisms. However, regarding rhythm prognosis, it is generally considered that sinus node dysfunction predisposes patients to the development of atrial tachyarrhythmia, particularly in combination with chronic pressure load and extensive suture lines in the right atrium, followed by formation of scar tissue. ${ }^{13-15,17}$

Currently, sinus node dysfunction per se is known to affect P-wave characteristics. Sinus node dysfunction with prolonged intra-atrial and interatrial conduction times has been observed to cause abnormalities in the morphology and duration of the P-waves. ${ }^{2}$ Also, P-wave characteristics were found to be good markers for predicting atrial tachyarrhythmia in patients with sinus node dysfunction. ${ }^{3}$ The high prevalence of sinus node dysfunction we observed in the IB group is consistent with the trend of P-wave characteristics, both of which suggest a risk of future atrial tachyarrhythmia. In contrast, we also noticed that sinus node dysfunction was common in the EC group; however, judging from the chronologic progress of the $\mathrm{P}$-wave characteristics, we anticipate that this cohort has a lower risk of atrial tachyarrhythmia. A longer duration of follow-up of these patients is clearly indicated.

\section{Study Limitations}

There were several important limitations to the study design. First, this was a retrospective study of a relatively small number of patients.

Second, the series was not concurrent because of important modifications of the surgical technique during the study period. This study was a comparative investigation based on different follow-up intervals. However, analysis of P-wave 
duration and dispersion with 10-year follow-up data showed a similar result: a tendency for P-wave duration and dispersion to increase in the APC and IB groups and decrease in the EC group.

Third, the 3 groups differed considerably in age at the time of the Fontan procedure. We have moved toward establishing the Fontan circulation at an earlier age (1-2 years) on the basis that earlier unloading of the single ventricle and elimination of cyanosis might have important effects on long-term performance of the ventricle and rhythm prognosis. ${ }^{6,17,24}$ Although there was no significant difference in preoperative $\mathrm{P}$-wave characteristics, in this study we did not address the effect of Fontan completion on rhythm prognosis at this younger age. However, when the analysis was performed with data only from patients less than 3 years old (4 in the APC group, 7 in the IB group, and 9 in the EC group), the results were similar.

Fourth, because cardiac morphology can affect P-wave characteristics, we limited the study population to patients with simple tricuspid atresia or stenosis, with the aim of obtaining a homogeneous morphologic background. Another reason for the patient selection in the study was the fact that the target anatomy for the Fontan procedure has clearly been expanded over time. In the 1970s and early 1980s, the most common anatomy was tricuspid atresia, whereas we now operate on patients with more complex anatomies. Therefore to compare the 3 types of Fontan procedures, we selected simple tricuspid atresia as the study population.

Finally, there was a potential methodological problem in the manual measurement of P-waves, which might have restricted the accuracy and reproducibility of the measurements. ${ }^{25}$ However, this method has been performed and accepted previously by other investigators.

\section{CONCLUSIONS}

After the IB procedure, patients commonly and increasingly experience prolonged $\mathrm{P}$-wave duration, large $\mathrm{P}$-wave dispersion, and sinus node dysfunction. This suggests that the IB procedure, although less progressive than the APC procedure, has the propensity to cause atrial tachyarrhythmias. In spite of a high prevalence of sinus node dysfunction after the EC procedure, the trend for relatively stable $\mathrm{P}$-wave characteristics over time supports the use of the EC procedure as the preferred option for the Fontan procedure.

We thank Dr Tom Wong, consultant cardiologist, Royal Brompton Hospital, and Mr Babulal Sethia, consultant cardiac surgeon, Royal Brompton Hospital, who gave us useful suggestions. We also thank Dr Bari Murtuza for assistance in preparing this manuscript.

\section{References}

1. Dilaveris PE, Gialafos EJ, Sideris SK, Theopistou AM, Andrikopoulos GK, Kyriakidis M, et al. Simple electrocardiographic markers for the prediction of paroxysmal idiopathic atrial fibrillation. Am Heart J. 1998;135:733-8.
2. Liu Z, Hayano M, Hirata T, Tsukahara K, Quin Y, Nakao K, et al. Abnormalities of electrocardiographic $\mathrm{P}$ wave morphology and their relation to electrophysiological parameters of the atrium in patients with sick sinus syndrome. Pacing Clin Electrophysiol. 1998;21:79-86.

3. De Sisti A, Leclercq JF, Stiubei M, Fiorello P, Halimi F, Attuel P. P wave duration and morphology predict atrial fibrillation recurrence in patients with sinus node dysfunction and atrial-based pacemaker. Pacing Clin Electrophysiol. 2002;25: 1546-54.

4. Wong T, Davlouros PA, Li W, Millington-Sanders C, Francis DP, Gatzoulis MA. Mechano-electrical interaction late after Fontan operation: relation between Pwave duration and dispersion, right atrial size, and atrial arrhythmias. Circulation. 2004; 109:2319-25.

5. Koh M, Yagihara T, Uemura H, Kagisaki K, Hagino I, Ishizaka T, et al. Optimal timing of the Fontan conversion: change in the P-wave characteristics precedes the onset of atrial tachyarrhythmias in patients with atriopulmonary connection. J Thorac Cardiovasc Surg. 2007;133:1295-302.

6. Uemura H, Yagihara T, Kawashima Y, Yamamoto F, Nishigaki K, Matsuki O, et al. What factors affect ventricular performance after a Fontan-type operation? J Thorac Cardiovasc Surg. 1995;110:405-15.

7. Adachi I, Yagihara T, Kagisaki K, Hagino I, Ishizaka T, Koh M, et al. Fontan operation with a viable and growing conduit using pedicled autologous pericardial roll: serial changes in conduit geometry. J Thorac Cardiovasc Surg. 2005;130: 1517-22.

8. van Son JA, Reddy M, Hanley FL. Extracardiac modification of the Fontan operation without use of prosthetic material. J Thorac Cardiovasc Surg. 1995;110: 1766-8.

9. Uemura H, Yagihara T, Yamashita K, Ishizaka T, Yoshizumi K, Kawahira Y. Establishment of total cavopulmonary connection without use of cardiopulmonary bypass. Eur J Cardiothorac Surg. 1998;1:504-7.

10. Stein E. Basic electrocardiology. In: Rapid analysis of electrocardiograms. Philadelphia: Lippincott, Williams \& Wilkins; 2000.

11. Garson A Jr. Electrocardiography. In: Garson A Jr, Bricker TJ, McNamara DG, eds. The science and practice of pediatric cardiology. Philadelphia: Lea \& Febiger; 1990.

12. Davignon A, Rautaharju P, Boisselle E, Soumis F, Megelas M, Choquette A. Normal ECG standards for infants and children. Pediatr Cardiol. 1979;1:123-31.

13. Kavey RE, Gaum WE, Byrum CJ, Smith FC, Kveselis DA. Loss of sinus rhythm after total cavopulmonary connection. Circulation. 1995;92(suppl): II304-8.

14. Manning PB, Mayer JE Jr, Wernovsky G, Fishberger SB, Walsh EP. Staged operation to Fontan increases the incidence of sinoatrial node dysfunction. J Thorac Cardiovasc Surg. 1996;111:833-9.

15. Cohen MI, Wernovsky G, Vetter VL, Wieand TS, Gaynor JW, Jacobs ML, et al. Sinus node function after a systematically staged Fontan procedure. Circulation. 1998;98(suppl):II352-8.

16. de Leval MR, Kilner P, Gewillig M, Bull C. Total cavopulmonary connection: a logical alternative to atriopulmonary connection for complex Fontan operations. Experimental studies and early clinical experience. J Thorac Cardiovasc Surg. 1988;96:682-95.

17. Fishberger SB, Wernovsky G, Gentles TL, Gauvreau K, Burnett J, Mayer JE Jr, et al. Factors that influence the development of atrial flutter after the Fontan operation. J Thorac Cardiovasc Surg. 1997;113:80-6.

18. Balaji S, Gewillig M, Bull C, de Leval MR, Deanfield JE. Arrhythmias after the Fontan procedure. Comparison of total cavopulmonary connection and atriopulmonary connection. Circulation. 1991;84(suppl):III162-7.

19. Gialafos EJ, Dilaveris PE, Synetos AG, Tsolakidis GF, Papaioannou TG, Andrikopoulos GK, et al. P wave analysis indices in young healthy men: data from the digital electrocardiographic study in Hellenic Air Force Servicemen (DEHAS). Pacing Clin Electrophysiol. 2003;26:367-72.

20. Li W, Xiao HB, Henein MY, Somerville J, Gibson DG. Progressive ECG changes before the onset of atrial flutter in adult congenital heart disease patients. Heart. 2001;85:703.

21. Ohuchi H, Hasegawa S, Yasuda K, Yamada O, Ono Y, Echigo S. Severely impaired cardiac autonomic nervous activity after the Fontan operation. Circulation. 2001;104:1513-8.

22. Davos CH, Francis DP, Leenarts MF, Yap SC, Li W, Davlouros PA, et al. Global impairment of cardiac autonomic nervous activity late after the Fontan operation. Circulation. 2003;108(suppl):II180-5.

23. Blaufox AD, Sleeper LA, Bradley DJ, Breitbart RE, Hordof A, Kanter RJ, et al. Functional status, heart rate, and rhythm abnormalities in 521 Fontan patients 6 to 18 years of age. J Thorac Cardiovasc Surg. 2008;136:100-7. 
24. Uemura H, Yagihara T, Kawahira Y, Yoshikawa Y, Kitamura S. Total cavopulmonary connection in children with body weight less than $10 \mathrm{~kg}$. Eur J Cardiothorac Surg. 2000;17:543-9.

25. Dilaveris PE, Gialafos EJ, Chrissos D, Andrikopoulos GK, Richter DJ, Lazaki E, et al. Detection of hypertensive patients at risk for paroxysmal atrial fibrillation during sinus rhythm by computer-assisted $\mathrm{P}$ wave analysis. J Hypertens. 1999; 17:1463-70.

\section{Discussion}

Dr Charles B. Huddleston (St Louis, Mo). This study is remarkable in a couple of respects. One is that the single-ventricle diagnosis was the same for all study groups. Given the heterogeneity of all patients that end up going through the Fontan pathway, that has eliminated some of the confounding factors in the analysis by focusing on this one diagnostic group.

The other interesting aspect, or the remarkable aspect, about this study is that it took someone, perhaps you, Dr Koh, to go through the mind-numbing task of reviewing hundreds of electrocardiograms measuring these $\mathrm{P}$-wave durations and dispersions because I do not know that there is an automated way to do that. When I spoke with our cardiologists about it, I was informed that it could be done only by hand. Therefore you can imagine the time involved in that particular task.

I do not think anybody is surprised that there would be this finding about the APC type of Fontan procedure given what we know about the incidence of atrial arrhythmias after that particular modification of the Fontan operation.

I think that the other confusing part about this is that the duration of follow-up plays a major role in the incidence of arrhythmias found in these patients. No specific arrhythmias were found in the intracardiac baffle group. There was just this concern based on the measurements of the P-wave duration and dispersion that raised further concerns about the possibility of arrhythmia development in the future.

Therefore we really are trying to decide as a group, I suppose, or perhaps individually, whether the intracardiac baffle or the extracardiac Fontan procedure are the better of the 2 options. I came to this meeting with my own preconceived notions, listened to the program at the postgraduate course yesterday, began to think that maybe things should be done differently at our center, heard this, and now I am back to where I was before. Therefore I am not quite sure where we should be going with all this.

The question that I have for you really is just one about this study. Specifically, where is the suture line placed for the intracardiac baffle? At least in the research we did in our laboratory, that was a critical aspect of our study, at least in the acute setting, as to how the atrial conduction was affected by that particular type of technique. You mentioned that you placed the suture lines for this baffle on the anterior aspect of the atrium, but could you relate that a little bit to where you make the atriotomy and the linea terminalis of the right atria?

Thank you very much. It was a very good presentation.

Dr Koh. Thank you, Dr Huddleston.

In the IB procedure, first we made an ordinary right atriotomy from the tip of the appendage toward the inferior vena cava parallel to the atrioventricular groove. Then we put the looser edge of the baffle patch along the inferior vena caval orifice and then up to the line parallel to the atriotomy. Then we connected the tip of the appendage to the pulmonary artery together with the superior edge of the patch. Finally, we closed the incision line together with the other lateral edge of the patch. Therefore in the IB procedure we have 4 suture lines: one is along the inferior vena caval orifice, 2 parallel lines are on the anterior aspect of the right atrial wall, and another line is on the anastomosis site between the appendage and the pulmonary artery. None of these lines are crossing the terminal crista. The sinus node was placed in the low-pressure atrial chamber.

Dr Carl L. Backer (Chicago, Ill). First, I want to congratulate you on a great study. In particular, this information is quite useful in patients who are candidates for a Fontan conversion. The P-wave analysis, as you previously reported, can be a predictor of when these patients are going to have atrial arrhythmias and become a candidate for Fontan conversion operation.

The question I wanted to focus on here was that I noticed that you had a relatively high incidence of sinus node dysfunction after the Fontan procedure. I think that if you critically look at patients who have had any of the Fontan-type procedures, sinus node dysfunction does develop, as happened in your series.

We have been proactive in this area, working in collaboration with our electrophysiologists. At the time of the primary Fontan procedure, we now place an epicardial atrial pacing lead, which we might or might not use in the perioperative period. However, over a period of time, many of those patients have had sinus node dysfunction, and we have then gone back and simply put in a pacemaker generator. You already have the lead, and therefore you do not have to open up the sternum again.

Given the high incidence of sinus node dysfunction in your series, what do you think about our strategy of routinely placing a prophylactic atrial epicardial pacing lead at the time of the primary Fontan procedure?

Dr Koh. We do not use permanent epicardial pacing leads on a routine basis. None of the patients in our center have had prophylactic pacemaker implantation at the time of the Fontan procedure. All patients who had sinus node dysfunction in the IB and EC groups in this series have been asymptomatic and free from atrial tachyarrhythmia thus far. I am personally reluctant to put a permanent pacing system in asymptomatic patients. I can agree that pacing can suppress atrial tachyarrhythmias, but there is a controversy about whether pacing is beneficial to the Fontan circulation. 